\title{
El monitoreo a mbulatorio de las contracciones uterinas no es útil para predecir parto prematuro
}

Frecuency of uterine contractions and the risk of spontaneous preterm delivery. lams JD, Newman RB, Thom EA, Goldemberg RL, y cols. N Engl J Med 2002; 346; 250-5

\section{Objetivo}

Evaluar la utilidad de las contracciones uterinas como predictoras de parto prematuro.

\section{Diseño}

Estudio observacional prospectivo

\section{Lugar}

Estudio multicéntrico realizado en 11 hospitales de EE.UU. entre 1994 y 1996.

\section{Pacientes}

Se incluyeron 306 mujeres entre 22 y 24 semanas de gestación. La mayoría, 254 pacientes, presentaban riesgo elevado para parto prematuro (parto prematuro previo ó metrorragia del segundo trimestre en el embarazo actual). Las restantes eran pacientes sín factores de riesgo.

\section{Evaluación de Factores Pronósticos}

Las pacientes fueron instruidas en la utilización de un monitor de contracciones y en la transmisión de los datos, que se realizó telefónicamente. Registraron la frecuencia de contracciones dos veces por día, dos días por semana desde su inclusión hasta el parto ó las 37 semanas. Se evaluaron además otras variables que pudieran afectar la frecuencia de las contracciones como la edad gestacional, el momento del día y el antecedente de parto prematuro.

\section{Medición de resultados}

Se comparó la frecuencia de contracciones de las mujeres que tuvieron el parto después de las 35 semanas con las que tuvieron el parto entre las 29 y 32,6 semanas y entre las 33 y 34,6 semanas. La máxima frecuencia de contracciones durante el día y durante la noche a cada edad gestacional fue evaluada como predictores de parto prematuro. Se determinó y comparó la sensibilidad, especificidad y valores predictivos de la frecuencia de contracciones con otros marcadores de riesgo como la evaluación digital y ecográ- fica del cuello uterino, la presencia o ausencia de fibronectina en la secreción cervical y el puntaje de Bishop (puntaje de variables compuestas que incluyen longitud cervical, dilatación, posición, consistencia y grado de descenso del feto).

\section{Resultados principales}

Un $35 \%$ de las pacientes tuvieron parto prematuro (< 37 semanas), $16 \%$ antes de las 35 y $6 \%$ antes de las 32 . La frecuencia de contracciones no tuvo relación con el antecedente de parto prematuro. La frecuencia de contracciones aumentó con la edad gestacional y por la noche. Las pacientes que tuvieron parto prematuro antes de la semana 35 tuvieron mayor frecuencia de contracciones tanto durante el día como durante la noche entre las 24 y 33 semanas. En el análisis multivariado* se detectó un aumento significativo de riesgo para parto prematuro con: la máxima frecuencia de contracciones nocturna solo entre las 27 y 28 semanas, la longitud cervical entre las 22 y 33 semanas, el puntaje de Bishop entre las 22 y 24 semanas y la presencia de fibronectina entre las 31 y 32 semanas. El valor predictivo positivo* del aumento de las contracciones en las distintas semanas estuvo entre el 25 y el $30 \%$. El valor predictivo negativo* de todas las variables estuvo entre el 80 y el $90 \%$.

Tabla. Riesgo de parto prematuro con ajuste multivariado

\begin{tabular}{l|c|c|c}
\multirow{2}{*}{ Prueba } & \multicolumn{3}{|c}{ Odds Ratio (IC 95\%) } \\
\cline { 2 - 4 } & 22-24 semanas & 27-28 semanas & 31-32 semanas \\
\hline Máximas contracciónes nocturnas & $3(0.6-14.6)$ & $3(1-8.6)$ & $1.3(0.3-5.2)$ \\
\hline Máximas contracciones diurnas & $3(0.3-33.6)$ & $1.6(0.4-6.2)$ & $0.5(0.1-3.2)$ \\
\hline Fibronectina & $2.3(0.6-8.2)$ & $2(0.6-6.6)$ & $3.8(1.1-13.2)$ \\
\hline Longitud cervical menor de 25mm $5.9(12.6-13.7)$ & $4(1.6-10.6)$ & $7.5(1.6-29.9)$ \\
\hline Puntaje de Bishop >4 & $4.3(1.6-10.6)$ & $1.7(0.7-4.6)$ & $3(0.8-12.7)$ \\
\hline
\end{tabular}

\section{Conclusión}

Aunque ninguna de las pruebas mostró una buena sensibilidad ${ }^{\star} y$ un alto valor predictivo positivo*, la frecuencia de contracciones resultó particularmente débil como predictora de parto prematuro.

Fuente de financiamiento: National Institute of Child Health and Human Development, EE.UU.

\section{Comentario}

El aumento de la frecuencia de contracciones uterinas conlleva al trabajo de parto y parto prematuro por lo que se han planteado múltiples estrategias para detectar y prevenir esta situación con el fin de reducir la tasa de prematurez. Los hallazgos del presente trabajo brindan una explicación de los estudios clínicos aleatorizados previos que no pudieron demostrar un efecto beneficioso del monitoreo en el hogar de las contracciones uterinas sobre la incidencia de parto prematuro, peso al nacimiento o mortalidad perinatal ${ }^{123}$

Tanto la frecuencia de contracciones, la longitud cervical, la presencia de fibronectina y el puntaje de Bishop presentan baja sensibilidad* y valor predictivo positivo* con un alto valor predictivo negativo*, por lo cual no son clínicamente útiles como pruebas de detección en pacientes asintomáticas. Su uso estaría limitado a evitar intervenciones innecesarias.

La valoración de la fibronectina y la longitud cervical han sido reportadas como útiles para descartar trabajo de parto prematuro en pacientes sintomáticas, grupo que no fue evaluado primariamente en este trabajo ${ }^{45}$.

Conclusión de los comentadores: Los resultados de este estudio indican que el monitoreo ambulatorio de las contracciones no sirve para identificar a las pacientes en riesgo de parto prematuro y por consiguiente no es útil para prevenir el parto prematuro en la práctica clínica. La búsqueda de mejores predictores de parto prematuro tiene como objetivo identificar grupos de embarazadas de mayor riesgo de parto prematuro para la evaluación, en estudios aleatorizados, de posibles intervenciones.

\section{Dra. Matelda Lisdero [ Becaria de Medicina Materno-Fetal ] \\ Dr. Lucas Otaño, Jefe [ Hospital Italiano de Buenos Aires ]}

\section{Referencias}

1.Grimes DA, Schultz KF. Randomized controlled trials of home uterine activity monitoring: a review and critique.Obstet Gynecol 1992;79:137-42

2.Sachs BP, Hellerstein S, FreemanR, Frigoleto F, HauthJC. Home monitoring of uterine activity: does it prevent prematurity? N Engl J Med 1991;325; 1374-7

3. Preventive Service Task Force. Home uterine activity monitoring for preterm labor. JAMA 1993; 270; 371-6

4. Leitich H, Brunbauer M, Kaider A, Egarter C, Husslein P. Cervical length and dilation of the internal cervical os detected by vaginal ultrasonography as marker for preterm delivery; 4. Leitich H, Brunbauer M, Kaider A, Egarter C, Husslein P. Cervace
a systematic review. Am J Obstet Gynecol 1999; 181; 1465-72

5.American College of Obstetricians and Gynecologists. ACOG Practice Bulletin: assessment of risk factors for preterm birth: clinical management guidelines for obstetricians-gynecologists. N ${ }^{\circ} 31$, October 2001. Obstet Gynecol 2001,98:709-16 